\title{
Feedback como Estratégia de Aprendizado no Ensino Médico
}

\author{
Feedback as a Teaching/Learning Strategy \\ in Medical Education
}

\author{
Angélica Maria Bicudo Zeferino ${ }^{1}$ \\ Rosângela Curvo Leite Domingues ${ }^{1}$
}

Eliana $\mathrm{Amaral}^{1}$

PALAVRAS-CHAVE:

- Feedback Psicológico;

- Ensino;

- Aprendizagem;

- Aptidão.

KEY-WORDS:

- Feedback;

- Teaching;

-Learning;

- Aptitude.

Recebido em: 18/07/2006

Aprovado em: 14/03/2007

\section{ABSTRACT}

Give feedback to the students is an important aspect in the learning process and an essential role of the teachers. In the context of medical education, feedback refers to information describing the students' performance in a given situation or activity. The ability on giving and receiving feedback improves learning outcomes since it provides a basis for self-directed learning and critical thinking, helps the student correct errors, reinforces desirable attitudes and shows the student how he can improve. Despite the patent lack of feedback throughout the medical course, students greatly desire and value this constructive tool and consider it as an important aspect of quality teaching. Effective feedback should be assertive, delivered with respect, descriptive, timely and linked to specific observed behaviors. Both faculty and students should be offered proper training in order to enhance their ability to give and receive feedback. Program directors should value the role of feedback as an essential teaching/learning strategy for the undergraduate curriculum. 
O conceito de feedback, informações usadas pelo sistema para seu ajuste, foi definido por engenheiros ${ }^{1}$. Na área educacional, feedback refere-se à informação dada ao aluno que descreve e discute seu desempenho em determinada situação ou atividade. O feedback gera uma conscientização valiosa para a aprendizagem, pois ressalta as dissonâncias entre o resultado pretendido e o real, incentivando a mudança; também aponta os comportamentos adequados, motivando o indivíduo a repetir o acerto $^{2}$. Se a informação for capaz de causar mudança no padrão de desempenho observado, teremos um processo de aprendizagem ${ }^{1}$.

Entre os princípios ligados à aprendizagem dos adultos, inclui-se o desejo de receber feedback, juntamente com a necessidade de participação ativa no processo de aprender, a autodiretividade e a curiosidade de explorar soluções ${ }^{2,3}$. Uma vez que reforça a prática reflexiva, o feedback está fortemente vinculado ao conceito de desenvolvimento profissional contínuo, habilidade considerada essencial no médico que se deseja formar ${ }^{4}$. A falta de feedback gera incertezas, amplifica o sentimento de inadequação e distancia o aluno dos objetivos propostos, podendo levá-lo a interpretar seus comportamentos de maneira totalmente inapropriada e a desenvolver uma "falsa confiança" ou medo exagerado do erro ${ }^{1,5}$. Criar oportunidades de receber feedback no início da prática clínica permite que o aluno desenvolva e aprimore sua autopercepção e contribui para que ele aprenda a se auto-avaliar de maneira coerente e precisa 1 .

No contexto da educação médica, em particular na área clínica, a habilidade de dar e receber feedback com eficácia tem uma importância singular e se mostrou efetiva em melhorar o desempenho ${ }^{2,5,6}$. O feedback contribui com a prática reflexiva, ou capacidade do profissional de rever suas próprias conclusões, raciocínio e decisões, uma habilidade essencial para desenvolver profissionalismo e aprimorar as habilidades cognitivas, essenciais na prática clínica ${ }^{5,7,8}$.

É um ingrediente essencial para a aprendizagem clínica, em especial quando fornecido imediatamente após o desempenho observado, como, por exemplo, na avaliação formativa ou somativa ${ }^{3}$. Estudos demonstram que o feedback está associado à melhoria do desempenho do aluno no exame físico e na prática clínica como um todo9,10.

Os alunos consideram o feedback uma importante ferramenta de aprendizagem e valorizam os professores que sabem fornecê-lo de maneira eficaz ${ }^{11}$. Num estudo com 3 mil alunos de último ano de Medicina e residentes, 95\% destes consideraram que dar e receber feedback de forma eficaz é a segunda entre 36 habilidades de ensino, sendo ultrapassada apenas pela capacidade de estar aberto para perguntas ${ }^{12}$.

\section{COMO DAR FEEDBACK DE FORMA EFICAZ}

Dar feedback exige habilidade, compreensão do processo, criação de um ambiente propício e de uma relação de confiança ${ }^{13}$. Não há como informar ao aluno que a sua hipótese diagnóstica estava errada ou que ele não colheu todos os dados necessários durante a história clínica sem causar uma sensação de desapontamento ou frustração. Por outro lado, essa informação é essencial e não pode ser omitida.

A validade do processo de feedback inicia-se na qualidade das observações feitas, e o observador deve estar comprometido com o processo, com o aluno e com a instituição. Além disso, deve utilizar um instrumento confiável de registro e seguir padrões bem estabelecidos de competência clínica ${ }^{1}$. A preparação dos professores para utilizar a ferramenta é essencial, incluindo a maneira como as informações devem ser transmitidas, oralmente ou por escrito ${ }^{14,15}$.

A maior eficácia do feedback só se consegue quando este enfoca, além de competências, as atitudes e outras dimensões do profissionalismo, repete-se em diferentes momentos do curso médico e quando o professor se preocupa em observar também as reações emocionais do aprendiz, estabelece os objetivos do aprendizado e formas de interação em consenso com o aluno e permite que este desenvolva seu raciocínio5,16.

As múltiplas tarefas devidas ao professor no aprendizado da prática clínica parecem contribuir para limitar o tempo dedicado a esse recurso didático. Salermo et al. ${ }^{14}$ encontraram que $75 \%$ do tempo foram utilizados na apresentação e discussão do caso e que o feedback, com muitas informações negativas ou pouco específicas, ocupou apenas 30 segundos. A falta de treinamento, o desejo de não ofender e a vontade de manter a auto-estima dos alunos são outras causas citadas para justificar o pouco uso da estratégia ${ }^{15}$.

Aprender a partir do feedback requer que este seja fornecido de forma construtiva e positiva, colaborando para que o aluno reflita criticamente e elabore um plano de melhoria em prática ${ }^{2,5}$. Uma boa estratégia é a utilização da "técnica do sanduíche", na qual o feedback sobre comportamentos inadequados é fornecido entre duas ações ou comportamentos positivos; essa estratégia é particularmente útil para os alunos considerados mais resistentes ${ }^{17}$. A efetividade do feedback é maior quando é:

(1) Assertivo. A comunicação dever ser clara, objetiva e direta. Por temer o impacto das palavras, o professor pode não ser direto, falando de forma vaga, com afirmações ambíguas que ofuscam a mensagem principal. O aluno, temendo uma avaliação negativa, não procura esclarecimentos, reforçando a falta de clareza do professor. Como resultado, apesar das intenções educati- 
vas, pouco é transmitido 5 . Assim, recomenda-se descrever os impactos e conseqüências de determinado comportamento, positivos ou negativos, assim como sugerir comportamentos alternativos ${ }^{18}$.

(2) Respeitoso. Este é um elemento fundamental para o sucesso do feedback, independentemente das diferenças de conhecimento, experiência, hierarquia ou características pessoais entre os interlocutores. Como é um processo compartilhado, docente e aluno devem encontrar pontos de concordância sobre os comportamentos que devem ser trabalhados; entender e respeitar a opinião do outro geram o ambiente de respeito para um feedback construtivo ${ }^{5}$.

(3) Descritivo. Embora o estudante, em geral, esteja ávido por ouvir a opinião dos professores, sua reação é menos resistente quando as palavras descrevem determinado comportamento ou ação, ao invés de julgá-lo ${ }^{3,5}$.

(4)Oportuno. O momento e o local para dar feedback ao aluno devem ser adequados, preferencialmente logo após a observação do comportamento e em ambiente reservado ${ }^{2}$.

(5) Específico. É fundamental que o docente indique claramente os comportamentos nos quais o aluno está tendo bom desempenho e aqueles nos quais o aluno pode melhorar. Exemplos e revisão dos fatos ocorridos contribuem para que o aluno reflita honestamente sobre seu desempenho' ${ }^{13}$.

\section{IMPLICAÇÕES PARA O ENSINO MÉDICO}

O uso do processo de feedback emerge como um componente essencial no desenvolvimento da prática reflexiva e da construção do profissionalismo. Apesar de sabidamente eficaz, não tem sido uma estratégia pedagógica utilizada rotineiramente no curso médico, por vezes sendo visto como um "ato de coragem" do professor ou como um "ato de tortura" para o aluno. Para sua implementação bem-sucedida e eficaz, todos os envolvidos devem compreender o processo de feedback e aceitá-lo como uma oportunidade de crescimento pessoal e profissional, uma ferramenta de desenvolvimento de carreira.

Os docentes devem ser estimulados a praticar o feedback como estratégia regular de ensino-aprendizagem, inserindoo precocemente na rotina de avaliação. Os alunos vão gradativamente se conscientizando do seu nível de competência e buscando aprimoramento, processos essenciais no desenvolvimento da prática reflexiva. Assim, o feedback pode ser incorporado de forma natural, tornando o ambiente de ensino seguro e os alunos menos hesitantes em aceitarem as críticas e sugestões. E, ao aceitá-las, mais facilmente se tornarão agentes ativos no processo de aprender, buscando se capacitar em suas deficiências.

Para contribuir neste processo, os alunos devem ser encorajados e treinados para receber, mas também realizar feedback, em relação a si mesmos e aos colegas. Um exemplo de estratégia nesta direção é solicitar que o aluno analise o vídeo de uma consulta feita pelo colega, guiado por um instrumento estruturado. Em seguida, deve apresentar o feedback por escrito ao colega, reforçando os comportamentos que não precisam ser modificados e identificando os que devem ser mudados. Também deve preparar uma auto-avaliação, que seria, posteriormente, comparada com o feedback que recebeu de seu par e de seu tutor. Essa atividade seria acompanhada e orientada por um docente e utilizada tanto para comentar e corrigir os comportamentos observados na consulta, como para avaliar o feedback fornecido. Dessa forma, os alunos estariam gradativamente construindo e praticando suas habilidades de observar, ouvir atentamente, perguntar e se expressar com clareza, elementos essenciais e indispensáveis também na relação com os pacientes e familiares.

Mas, para essa mudança ocorrer, é preciso disponibilizar aos professores um conjunto de práticas e informações que aprimorem sua habilidade de fornecer feedback e de utilizá-lo como estratégia de ensino. Os programas de desenvolvimento docente para o ensino médico devem incluir tal componente. Há evidências de que programas de desenvolvimento são amplamente aceitos pelos docentes e apresentam efeitos positivos e que a habilidade de feedback está entre os temas sugeridos em sua composição ${ }^{19}$. Demonstrou-se que os professores apresentaram melhoria significativa, embora modesta, em sua habilidade de dar feedback após terem participado de três seminários com duração de 90 minutos e que este tema deve ser constantemente revisto na capacitação docente ${ }^{14}$. Um exercício prático de avaliação das habilidades do professor como facilitador do aprendizado, seguida de feedback, pode ser uma vivência interessante que exemplifica o potencial dessa estratégia de aprendizagem.

A arte de ensinar e de aprender, visando à qualidade do desempenho clínico, pode indubitavelmente ser aprimorada com a prática do feedback regular. Neste artigo, buscou-se auxiliar os educadores médicos a pensarem criticamente sobre como incorporar ou aprimorar a habilidade de fornecer feedback. Reproduzindo o processo de incorporação de outras tecnologias, procuramos fornecer elementos teóricos para o entendimento da proposta, apresentando algumas evidências de sua efetividade. 


\section{REFERÊNCIAS}

1. Ende J. Feedback in clinical medical education. JAMA. 1983; 250(6): 777-81.

2. Collins J. Education techniques for lifelong learning: principles of adult learning. Radiographics 2004; 24(5): 1483-9.

3. Rolfe IE, Sanson-Fisher RW. Translating learning principles into practice: a new strategy for learning clinical skills. Med Edu. 2002; 36(4): 345-52.

4. Rodgers KG, Manifold C. 360-degree feedback: possibilities for assessment of the ACGME core competences. Acad Emerg Med. 2002; 9(11): 1300 -4.

5. Henderson P, Ferguson-Smith, AC, Johnson, MH. Developing essential professional skills: a framework for teaching and learning about feedback. BMC Med Educ. [online]. 2005; 5 [capturado maio 2006]. Disponível em: http/ / www.biomedcentral.com/1 472-6920/5/11.

6. Veloski J, Boex JR, Grasberger MJ, Evans A, Wolfson DB. Systematic review of the literature on assessment, feedback and physicians' clinical performance: BEME guide n. 7. Med Teach. 2006; 28(2): 117-28.

7. Lachman N, Pawlina W. Integrating professionalism in early medical education: the theory and application of reflective practice in the anatomy curriculum. Clin Anat. 2006;19(5): 456-60.

8. Mamede S, Schmidt HG. Correlates of reflective practice in medicine. Adv Health Sci Educ Theory Pract. 2005; 10(4): 32737.

9. Newble DI, Jaeger K. The effect of assessment and examinations on the learning of medical students. Med Educ. 1983; 17(3): 165-71.

10. Papa FJ, Aldrich D, Schumacker RE. The effects of immediate online feedback upon dignostic performance. Acad Med 1999; 74(10Supl.): 16-8.

11. Rotenberg BW, Woodhouse RA, Gilbart M, Hutchison CR. A needs of assessment of surgical residents as teachers. Can J Surg. 2000; 43(4): 295-300.

12. Schultz KW, Kirby J, Delva D, Godwin M, Verma S, Birtwhistle R, et al. Medical students'and residents' preferred site characteristics and preceptor behaviours for learning in the ambulatory setting: a cross-sectional survey. BMC Med Educ. [online]. 2004; 4 [capturado abr. 2006] abril]. Disponível em: http:/ / www.biomedicentral.com/ 1472-6920/4/12.

13. Gordon J. ABC of learning and teaching in medicine: one to one teaching and feedback. BMJ. 2003; 326(7388): 543-5.
14. Salerno SM, O’Malley PG, Pangaro LN, Wheeler GA, Moores LK, Jackson JL. Faculty development seminars based on the one-minute preceptor improve feedback in the ambulatory setting. J Gen Inter Med. 2002; 17(10): 77987.

15. Dobbie A, Tysinger JW. Evidence-based strategies that help office-based teachers give effective feedback. Fam Med. 2005; 37(9): 617-9.

16. Menachery EP, Knight AM, Kolodner K, Wright SM. Physician characteristics associated with proficiency in feedback skills. J Gen Intern Med. 2006; 21(5): 440-6.

17. Lucas JH, Stallworth JR. Providing difficcult feedback: TIPS for the problem learner. Fam Med. 2003; 35(8): 544-6.

18. Jerome PJ. Coaching through effective feedback: a practical guide to successful communication. California: R. Chang Associates; c1994.

19. Kilminster SM, Jolly BC. Effective supervision in clinical practice settings: a literature review. Med Educ. 2000; 34(10): 827-40.

\section{Conflito de interesse}

Declarou não haver.

\section{Endereço para correspondência}

Angélica Maria Bicudo-Zeferino

Rua Madre Paulina, 66 - Condomínio Residencial Parque Rio das Pedras - 13085-150 - Campinas - SP

e-mail: ambz@fcm.unicamp.br 